\title{
LDL Subtype Pattern
}

National Cancer Institute

\section{Source}

National Cancer Institute. LDL Subtype Pattern. NCI Thesaurus. Code C120637.

A description of the low density lipoprotein particle pattern (an interpretation of the amounts of LDL particles based on size and density) in a sample. 